\title{
Influence of Smoothing Length and Virtual Particles on SPH Accuracy
}

\author{
T. El-Gammal, E. E. Khalil, H. Haridy, and E. Abo-Serie
}

\begin{abstract}
Using a well known 2-D transient heat conduction benchmark, a parametric study is conducted to identify the best values for the smoothing length (h) as function of particles spacing in order to minimize the error in the temperature distribution particularly at the center of the domain when applying the Smooth Particle Hydrodynamics (SPH) algorithm. Also the effect of the virtual particles on the numerical solution has been investigated. The results showed that the predefined smoothing length can have significant influence on the accuracy of the prediction and using the optimum value leads to minimum error in temperature prediction values. The results obtained with and without virtual particles has been presented and compared with the analytical solution.
\end{abstract}

Index Terms-Meshless, smoothing length, SPH, virtual particles.

\section{INTRODUCTION}

One of the most popular numerical meshless methods for solving the physical problems is the Smoothing Particle Hydrodynamics (SPH). It's a Lagrangian adaptive method that doesn't need to grid/mesh for solving the discretized domain. In this method the physical domain is discretized into unconnected particles that have physical properties (i.e. volume, mass, velocity....etc) of the domain [1]. Unlike the conventional numerical methods such as Finite Difference (F.D.) the meshless method uses field functions and their derivatives at different points (particles) whose values are approximately calculated using weighting (smoothing) kernel function. SPH is one of the recently developed meshless methods which have acquired the reliability and trustworthy in the last years such as the meshless element-free Galerkin (EFG) method [2]- [4], moving least-square methods [5], [6] and the diffuse approximation method [7].

The Lagrangian nature of SPH makes it more suitable for simulations involving fluid free surfaces, interfaces between different material, multiphase flow and highly deformable geometries. Simulating such applications by mesh based methods is challenging and required large memory and long computational time due to the continuous change in the computational mesh in space and time to fit the domain. After being developed for simulating the cosmological

Manuscript received November 30, 2012; revised February 2, 2013.

T. El-Gammal, E. E. Khalil, and H. Haridy are with the mechanical power engineering department, Cairo University, Giza, Egypt (e-mail: tarekelgammal@eng.cu.edu.eg; khalile1@asme.org; hatem_kayed@eng.cu. edu.eg).

E. Abo-Serie was with the mechanical power engineering department, Cairo University, Egypt. He is now with Mechatronics Department, Mevalana University, Konya, Turkey (e-mail: eaboserie@mevlana.edu.tr) phenomenon [8], [9], SPH has been used in solving fluid and solid mechanics problems.

The main advantages of SPH are the easiness to model complex material surface behavior and complicated physics, such as solidification [10], [11] and multiphase flows [12].

In SPH, The field function (Temperature, pressure, velocity....etc.) is calculated for a discrete particle from the field functions of the all surrounding particles weighted by the smoothing function. The smoothing (weighting) kernel function takes into account the effect of the neighboring particles within a predefined radius called smoothing length (h). Many famous kernel functions use a dependent factor (K) to multiply with the smoothing length (h).

In this work the value of smooth length has been varied to investigate its effect on the final result. The problem chosen for this investigation is a 2-D diffusion transient case which has been simulated previously using SPH technique with a constant predefined smooth length [13]. This previous study showed significant error particularly at the location where low temperature gradient exist (at the center of the plate). The current study developed the technique to minimize the error by varying the smooth length and introducing virtual ghost particle to improve the boundary conditions representation. The results have been compared with previous investigation and the analytical solution of the conduction equation.

\section{PROBlem DEFINITION}

The problem used here is the famous benchmark 2-D rectangular plate of lengths $L x H$ undergoing heat conduction process. The process will be mainly cooling due to isothermal cooling boundaries. The plate temperature will cool from initial temperature $\left(T_{i}\right)$ to final temperature $\left(T_{f}\right)$ which depends on the final state required to be stopped at.

For 2-D heat diffusion in Cartesian coordinates, the equation for the temperature field $T=T(x, y, t)$ is

$$
\frac{\partial T}{\partial t}=\alpha\left(\frac{\partial^{2} T}{\partial x^{2}}+\frac{\partial^{2} T}{\partial y^{2}}\right)
$$

where $(\alpha)$ is the thermal diffusivity in $\left(\mathrm{m} / \mathrm{s}^{2}\right)$.

An analytical solution based on separation of variables and substitution of specified boundary conditions can be written in symbolic form to identify the temperature of the interior of the plate $T(x, y, t)$ as follows:

$$
\begin{aligned}
& T(x, y, t) \\
& =\frac{16 T_{i}}{\pi^{2}} \sum_{k=1,3, . .}^{\infty} \sum_{l=1,3, . .}^{\infty} \frac{e^{\left[-\alpha \pi^{2}\left(k^{2} / L^{2}+l^{2} / H^{2}\right) t\right]}}{k l} \sin \left(\frac{k \pi x}{L}\right) \sin \left(\frac{l \pi x}{H}\right)
\end{aligned}
$$




\section{MeTHODOLOGY}

SPH formulation starts from the integral representation of field function using the Dirac delta function $\delta\left(x_{i j}\right)$.The following relation states the one dimensional mathematical concept:

$$
f\left(x_{i}\right)=\int f\left(x_{j}\right) \delta\left(x_{i j}\right) d x \delta\left(x_{i j}\right)= \begin{cases}1 & i=j \\ 0 & \text { else }\end{cases}
$$

The main step of the derivation of SPH is substituting the Dirac delta function with even, limited-zone, kernel function $W\left(x_{i j}, h\right)$. Then we can write the function approximation in SPH formulation as:

$$
f\left(x_{i}\right) \approx\left\langle f\left(x_{i}\right)\right\rangle=\int f\left(x_{j}\right) W\left(x_{i j}, h\right) d x
$$

where $\left\langle f\left(x_{i}\right)\right\rangle$ is the kernel approximation of the scalar field $f(x)$ at particle $(i)$.

For two or three dimensions, the kernel function $W\left(x_{i j}, h\right)$ can be replaced by $W\left(r_{i j}, h\right)$ such that $\left(r_{i j}\right)$ is the vectorial distance between $(i)$ and $(j)$. Also the differential length $(d x)$ is updated to differential area $(d s)$ or volume $(d v)$.

The gradient $(\partial f(x i) / \partial x)$ and Laplacian $\left(\partial^{2} f(x i) / \partial x^{2}\right)$ of the field function are evaluated in integral representation[13] to be:

$$
\begin{aligned}
& \partial f\left(x_{i}\right) /_{\partial x} \approx\left\langle\nabla_{i} f(x)\right\rangle=\int f\left(x_{j}\right) \nabla_{i} W\left(x_{i j}, h\right) d x \\
& \partial^{2} f\left(x_{i}\right) / \partial x^{2} \approx\left\langle f_{x x_{i}}\right\rangle \\
& \quad=2 \int \frac{\left[f\left(x_{i}\right)-f\left(x_{j}\right)\right]}{r_{i j}^{2}} \cdot x_{i j} \cdot \nabla_{i} W\left(x_{i j}, h\right) d x
\end{aligned}
$$

The second main step of SPH is after the integral representation is the particle approximation. The field functions in (4), (5) and (6) are discretized in spaced, differential particles representing the domain, so the integrations approximate to summations of functions weighted by the kernel function.

$$
\begin{gathered}
\left\langle f\left(x_{i}\right)\right\rangle=\sum \frac{m_{j}}{\rho_{i}} f\left(x_{j}\right) W\left(r_{i j}, h\right) \\
\left\langle\nabla_{i} f(x)\right\rangle=\sum \frac{m_{j}}{\rho_{i}} f\left(x_{j}\right) \nabla_{i} W\left(r_{i j}, h\right) \\
\left\langle f_{x x_{i}}\right\rangle=2 \sum \frac{m_{j}}{\rho_{i}} \frac{\left[f\left(x_{i}\right)-f\left(x_{j}\right)\right]}{r_{i j}^{2}} \cdot x_{i j} \cdot \nabla_{i} W\left(r_{i j}, h\right)
\end{gathered}
$$

SPH formulation mentioned above can be substituted into the heat diffusion equation (1). Accordingly, the SPH representation of pure heat conduction case in 2-D can be written as follows:

$$
\begin{aligned}
\frac{\partial T}{\partial t}=2 \alpha\left(\sum \frac{m_{j}}{\rho_{i}}\right. & \frac{\left[T\left(x_{i}\right)-T\left(x_{j}\right)\right]}{r_{i j}^{2}} \cdot x_{i j} \cdot \nabla_{i} W \\
& \left.+\sum \frac{m_{j}}{\rho_{i}} \frac{\left[T\left(x_{i}\right)-T\left(x_{j}\right)\right]}{r_{i j}^{2}} \cdot y_{i j} \cdot \nabla_{i} W_{i j}\right)
\end{aligned}
$$

The compact cubic spline is kernel function has been used in this study. It was firstly proposed by Monaghan and

\section{Lattanzio [14].}

The formulations of the cubic spline kernel function $\left(W_{i j}\right)$ and its spatial derivative can be shown in (11) and (12).

$$
\begin{gathered}
W\left(r_{i j}, h\right)=W_{i j}=a_{n} *\left\{\begin{array}{lr}
\frac{2}{3}-R^{2}+\frac{1}{2} R^{3} & 0 \leq R<1 \\
\frac{1}{6}(2-R)^{3} & 1 \leq R<2 \\
0 & R>2
\end{array}\right. \\
\nabla_{i} W_{i j}=\frac{d W_{i j}}{d R} \times \frac{d R}{d r_{i j}} \times \frac{\left(x_{i}-x_{j}\right)}{r_{i j}}
\end{gathered}
$$

where $\left(R=r_{i j} / h\right)$ is the ratio between the magnitude of the distance between two neighboring particles $\left(r_{i j}\right)$ and the smoothing length $(h)$. In Eq. (11), $(n)$ is the dimension of the space, and the coefficient $\left(a_{n}\right)$ is dependent on the smoothing length $(h)$ and has values:

$$
a_{1}=\frac{1}{h} \quad a_{2}=\frac{15}{7 \pi h^{2}} \quad a_{3}=\frac{3}{2 \pi h^{3}}
$$

In one, two, and three dimensions, respectively.

\section{NUMERICAL ANALYSIS}

\section{A. Domain Particles Distribution}

Using Matlab ${ }^{\circledR}$, the plate domain $(L=H)$ is discretized into $41 \times 41$ particles which are classified into interior and boundary particles.

The initial temperature $\left(T_{i}\right)$ is set to all the particles. Particle differential area will be calculated from its spacings in $\mathrm{x}$ and $\mathrm{y}$ coordinates (i.e. $m / \rho=d s=d x \times d y$ ). The plate starts at $T_{i}=T(x, y, 0)=100{ }^{\circ} \mathrm{C}$ with isothermal cooling boundaries $T_{b}=T(0, y, T)=T(L, y, T)=T(x, 0, T)=T(x, H, T)$ $=0^{\circ} \mathrm{C}$.

\section{B. Virtual (ghost) Particles}

The virtual or ghost particles [15], [16] are generated in every time step as an image for the medium particles near the boundary (i.e. lie within the kernel domain of the wall/boundary particle) as in Fig. 1.

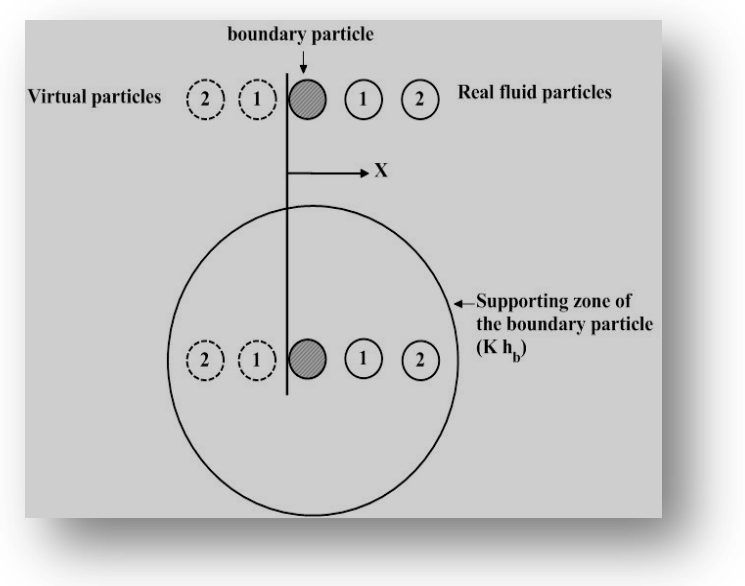

Fig. 1. Real and virtual particles within the supporting zone of the boundary particle

The virtual particle gains its physical properties from its real particle and the boundary condition of the wall. The artificially existed particles play an important role in completing the kernels of the particles to give accurate 
physical properties for all particles. Also they facilitate the applying of the boundary conditions as in F.D. especially for Neumann boundary condition.

In the problem domain, the virtual particles were applied to the four boundaries as shown in Fig. 2.

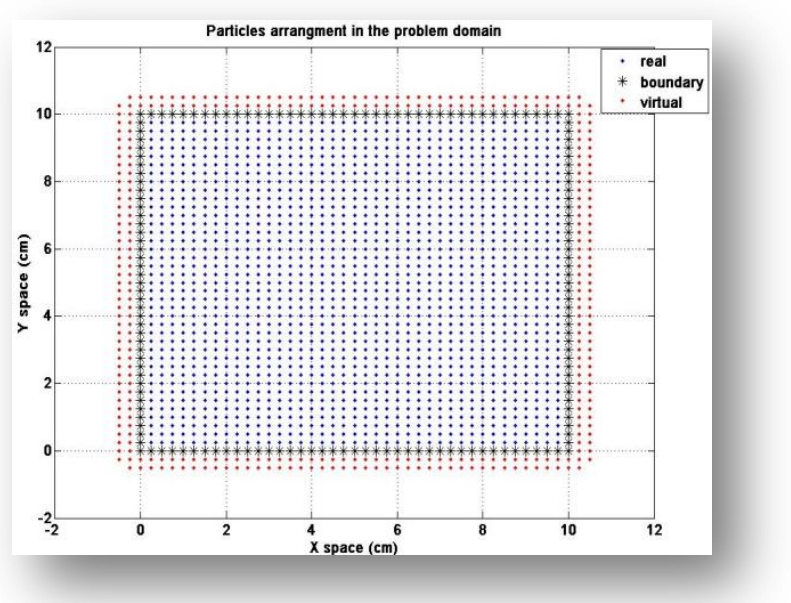

Fig. 2. Plate domain discretization into real, boundary and virtual particles

\section{Time Marching}

Depending on implicit second order Leapfrog method, the temperature at the mid of the current time step is calculated using the value of mid of the previous time step and the temperature rate of change based on the values of the time step preceding.

$$
\begin{aligned}
& \text { At } t=d t(\text { i.e. } n=1) \\
& \qquad T_{\frac{1}{2}}=T_{0}+\left.\left(\frac{d t}{2}\right) * \frac{\partial T}{\partial t}\right|_{n=1}
\end{aligned}
$$

where $\left.\frac{\partial T}{\partial t}\right|_{n=0}$ are evaluated by initial values $\mathrm{T}_{0}$ from the heat conduction equation.

For $\mathrm{n}>1$

$$
T_{(n-1)+\frac{1}{2}}=T_{(n-1)-\frac{1}{2}}+\left.d t * \frac{\partial T}{\partial t}\right|_{n}
$$

where $\left.\frac{\partial T}{\partial t}\right|_{n}$ are evaluated by $\mathrm{T}_{\mathrm{n}-1}$ from the heat conduction equation.

The time step has been chosen to satisfy the Monaghan and Cleary [17] criterion for pure heat conduction problems,

$$
d t \leq 0.15 \frac{h^{2}}{\alpha}
$$

In this case, the thermal diffusivity and time step were considered as $\left(\alpha=1 \mathrm{~cm}^{2} / \mathrm{s}, d t=0.001 \mathrm{~s}\right)$ respectively. The value of $(h)$ was then optimized to minimize the error.

In a second step, the optimized SPH algorithm was compared with no-virtual SPH to study the error due to the incomplete kernels near the boundary. Both results were taken at the same (hopt) and the time step $(d t=0.001 \mathrm{~s})$.

\section{RESULTS}

\section{A. Optimum Smoothing Length}

Fig. 3 lists the percentage errors $\left(\frac{T_{\text {analytical }}-T_{S P H}}{T i}\right)$ after $t=8 s$ for different smoothing lengths. The error varies throughout the plate and its maximum value is located in the center of the plate. The table shows clearly that there is an optimum value for smoothing length $(h o p t=1.28 d x)$ for best accuracy and the accuracy drops down for further increase or decrease in the value. Thismeans that $\left(h_{\text {opt }}\right)$ generates the best shape of kernel function to the temperature profile for the most of points (especially points close to the center). However, at the same time, the points near the boundary lose its accuracy if the virtual particles are not taken into considerations.

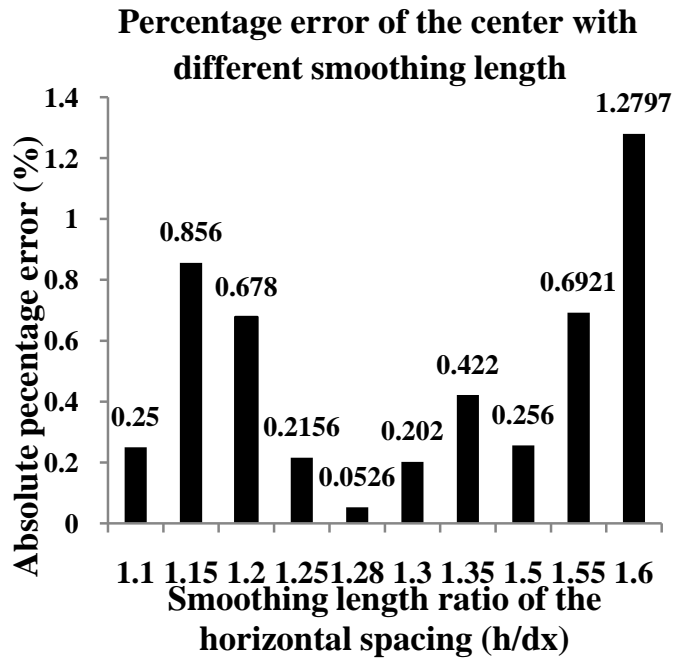

Fig. 3. The effect of changing the smoothing length on the accuracy of the SPH solution

After the choice of $\left(h_{o p t}\right)$, the results produced by the code are compared with conventional SPH results introduced by $\mathrm{R}$. Rook [13] to determine the effect of the virtual particles at $t=1,4,8 \mathrm{~s}$, the results have been compared with the reference obtained from the analytical solutions. The comparison is illustrated in isothermal contour lines shown in figures 4, 5, and 6 . The optimized smoothing length achieves better accuracy than the conventional SPH. This is obvious when time marches to the steady state. The conventional SPH error increases and spreads over the whole contour lines in a noticeable way. The main reason for that is the high gradient exists near the boundaries and diminishes towards the centers, so the increase or decrease of smoothing on particles of high gradients distorts the weighting kernel function and consequently the supporting area of a particle nearby boundary becomes greatly inaccurate.

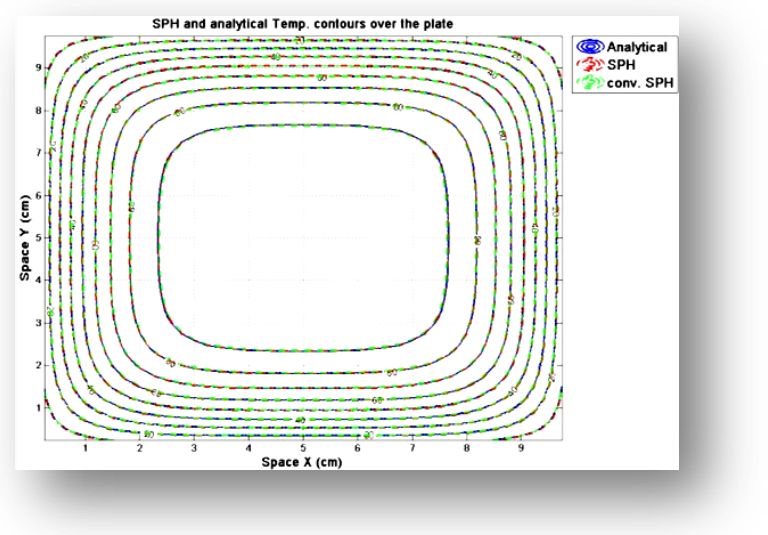

Fig. 4. Isothermal contour lines at $t=1 \mathrm{~s}$ 
Needless to say that adding the virtual particles to the conventional SPH leads to an increase in computational time. Including the virtual particles shows computational time of $31.82 \mathrm{~s}$ while the no-virtual SPH generates its results in 23.53 $s$. However, with the optimized smoothing length the computation time was less than that of the conventional SPH which took $87 s$.

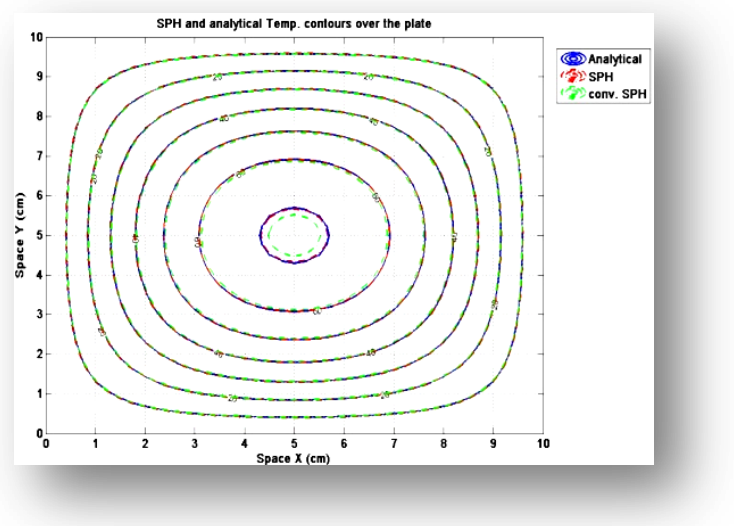

Fig. 5. Isothermal contour lines at $t=4 \mathrm{~s}$

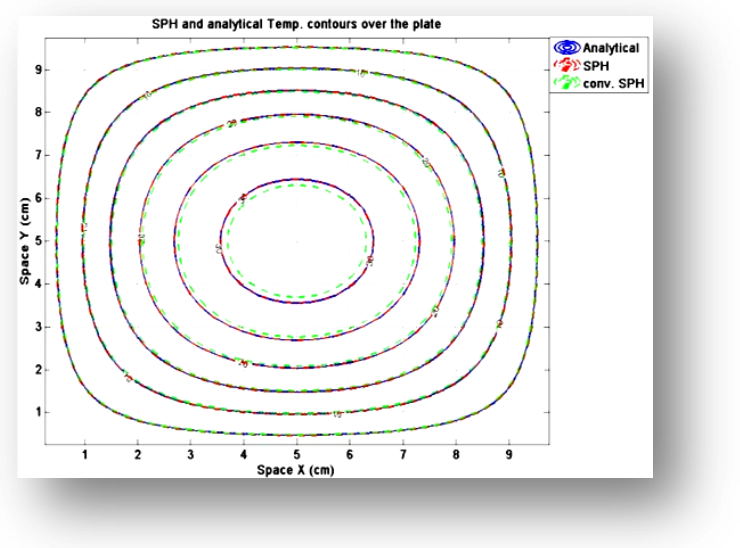

Fig. 6. Isothermal contour lines at $t=8 \mathrm{~s}$

\section{B. Virtual Particles}

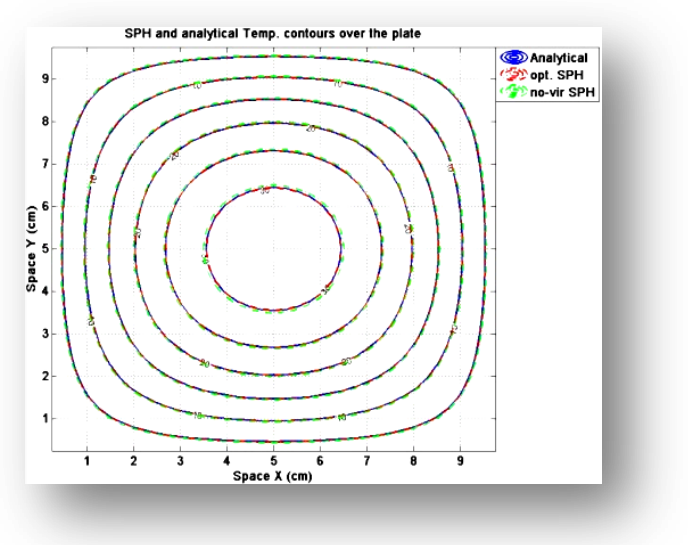

Fig. 7. Isothermal contour lines at $t=8 s$ (no-virtual SPH with optimized SPH)

After the determination of $\left(h_{\text {opt }}\right)$, the effect of virtual particles is compared with no-virtual SPH and the results are presented in Figs. 7 and 8 which show the contours lines of no-virtual SPH outfitting the analytical lines at the center but in lower behavior than conventional SPH because of optimized smoothing length used in it. No-virtual SPH missed the accuracy except in corners. In later times of cooling (i.e. $t>8 s$ ), the results of no-virtual SPH lose their accuracy as well as the conventional SPH. This is because the error of incomplete kernels is transmitted and increased among particles towards the center to reach a maximum value.

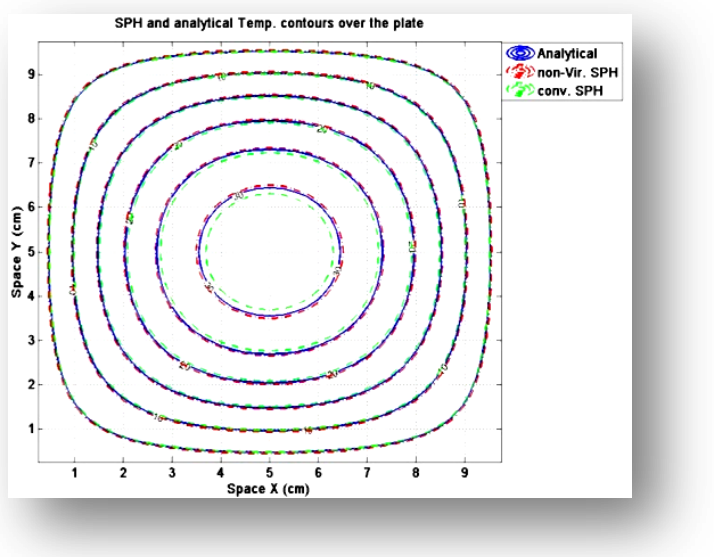

Fig. 9. Isothermal contour lines at $t=8 s$ (no-virtual SPH with conventional $\mathrm{SPH})$

\section{CONCLUSION}

Optimizing the smoothing length in SPH achieves the merits of high accuracy, consistent contours with the analytical solution, and less computation time. Any increase or decrease in the smoothing length will change the kernel weighting function $\left(W_{i j}\right)$ in (shape and value) and disorients it from the actual temperature profile. Although the smoothing length has been considered constant throughout the domain it is believed that having special distribution for the smoothing length can lead to more accurate results but that will be on the account of computation time.

\section{REFERENCES}

[1] G. R. Liu and M. B. Liu, Smoothed particle hydrodynamics: a meshfree particle method, Singapore: World Scientific Publishing Co., 2003 , ch. 1 , pp. 18

[2] I. V. Singh, "The numerical solution of heat transfer in extended surfaces using meshless element free galerkin method," Journal of Energy, Heat and Mass Transfer, vol. 25, pp. 135-155, 2003.

[3] I. V. Singh, K. Sandeep, and R. Prakash, "Heat transfer analysis of two dimensional fins using meshless element free galerkin method," Numerical Heat Transfer, Part A: Applications: An International Journal of Computation and Methodology, vol. 44, no.1, pp. 73-84, 2003.

[4] I. V. Singh and A. Singh, "A meshfree solution of two-dimensional potential flow problems," World Academy of Science, Engineering and Technology, 2009.

[5] Y. J. Dai, X. H. Wu, and W. Q. Tao, "Weighted least-squares collocation method (WLSCM) for 2-d and 3-d heat conduction problems in irregular domains," Numerical Heat Transfer, Part B. Fundamentals, vol. 59, no. 6, pp. 473-494, 2011

[6] J. Y. Tan, L. H. Liu, and B. X. Li, "Least-squares collocation meshless approach for coupled radiative and conductive heat transfer," Numerical Heat Transfer, Part B: Fundamentals: An International Journal of Computation and Methodology, vol. 49, no. 2, pp. 179-195, 2006.

[7] L.A. Dombrovsky, W. Lipinski, and A. Steinfeld, "A diffusion based approximate model for radiation heat transfer in a solar thermochemical reactor," Journal of Quantitative Spectroscopy \& Radiative Transfer, vol. 103, pp. 601-610, 2007.

[8] L. B. Lucy, "A numerical approach to the testing of the fission hypothesis,” Astro. J., vol. 82, pp. 1013-1024, 1977. 
[9] R. A. Gingold and J. J. Monaghan, "Smooth particle hydrodynamics: theory and application to non-spherical stars," Mon. Not. R. Astron. Soc., vol. 181, pp. 375, 1977.

[10] P. Cleary, J. Ha, V. Alguine, and T. Nguyen, "Flow modelling in casting processes," Applied Mathematical Modelling, vol. 26, pp. 171-190, 2002.

[11] J. J. Monaghan, H. E. Hupper, and M. G. Worster, "Solidification using smoothed particle hydrodynamics," Journal of Computational Physics, vol. 206, pp. 684-705, 2005.

[12] A. M. Tartakovsky, K. F. Ferris, and P. Meakin, "Lagrangian particle model for multiphase flows," Computer Physics Communications, vol. 180 , pp. $1874-1881,2009$.

[13] R. Rook, M.Yildiz, and S. Dost, "Modeling transient heat transfer using sph and implicit time integration," Numerical Heat Transfer, Part B: Fundamentals: An International Journal of Computation and Methodology, vol. 51, no.1, pp. 1-23, 2007.

[14] J. J.Monaghan and J. C. Lattanzio, "A refined particle method for astrophysical problems," Astronomy and Astrophysics Journal, vol. 149, pp. 135-143, 1985.

[15] F. A. Allahdadi, T. C. Carney, J. R. Hipp, L. D. Libersky, and A. G. Petschek, "High strain Lagrangian hydrodynamics: a three-dimensional SPH code for dynamic material response," Journal of Computational Physics, vol. 109, pp. 67-75, 1993.

[16] P. W. Randles and L. D. Libersky, "Smoothed particle hydrodynamics: some recent improvements and applications," Computer Methods in Applied Mechanics and Engineering, vol. 138, pp. 375-408, 1996.

[17] P. W. Cleary and J. J. Monaghan, "Conduction modelling using smoothed particle hydrodynamics," J. Comput. Phys., vol. 148, pp. 235-236, 1999.

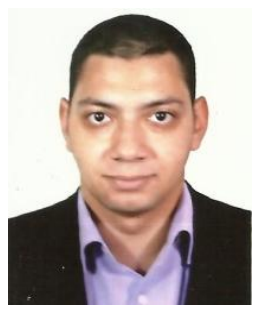

Tarek El-Gammal was born in Egypt in 1988. He was graduated with honors from Cairo University, Faculty of Engineering, Mechanical Power Department in 2010, He is currently working as a teaching assistant in Cairo University. His research interest is related to fluid flow problems, computational fluid dynamics and numerical methods

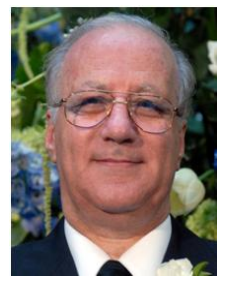

Essam Khalil was born in Scotland, in 1948. He Obtained his B.Sc. in Mechanical Engineering, Cairo University, Cairo, Egypt, 1971 with honors, M.Sc. in Mechanical Engineering, Cairo University, Cairo, Egypt, 1973. Prof. Khalil obtained his Ph.D. in Mechanical Engineering, London University ,London, United Kingdom in 1976. His major field of study is energy efficiency, Combustion and air conditioning. He was appointed at Cairo University, 1971 as teaching assistant and was assistant professor in 1977, associate professor in December 1982 Since June 1988 and till now he is Professor of Mechanical Engineering at Cairo University. He had published more than 525 Journal and International conference papers and 11 text and reference books. He is the Chair of the National Air Conditioning Code Committee and the Arab HVAC Code. His latest published books are entitled Air Conditioning of Hospitals and healthcare facilities, (Germany, Lap Lambert, 2012) and Boiler Furnace Design, (Germany, Lap Lambert, 2013). His research interests include combustion Chamber Design, energy and indoor air quality. Prof. Khalil is Fellow AIAA, Fellow ASME and Fellow ASHRAE; he is the elected Convenor ISO TC205WG2 on Energy Efficiency, Distinguished Lecturer ASHRAE since 2004. He is the Deputy Director (International (AIAA) and the Sub regional Chair for ASHRAE. He was awarded the Outstanding Achievement in Science Award, Cairo University, 2005,2007,2009,2010. Best Paper Award in Terrestrial Energy, AIAA 2005. In 2009 he received the ASME Westinghouse Gold Medal and Award, and in 2010 he was awarded the AIAA Energy Systems Award. In November 2012 he was awarded the ASME Gold James Harry Potter Award and Medal.

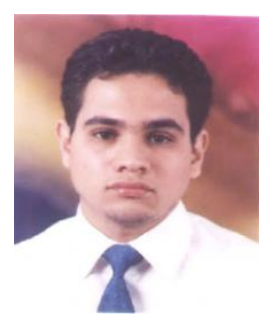

Hatem Haridy is an assistant Professor of Mechanical Power Engineering at Cairo University, where he has been since 2002. He also currently works as a part timer lecturer at "AUC" American University in Cairo and "BUE" British University in Egypt. He received a BSc from Cairo University in 2002, then MSc and Ph.D. was granted from the same university in 2006 and 2010 respectively.

His research interests are modeling of turbulent diffusion flames, spray and atomization and sustainable energy. He has worked on modeling a novel scheme of skewed two-dimensional probability density function of turbulent diffusion flames. This model can predict rim attached flames as well as unstable lifted flames. He is working on designing and local manufacturing of new generation of solar parabolic trough used in desalination units.

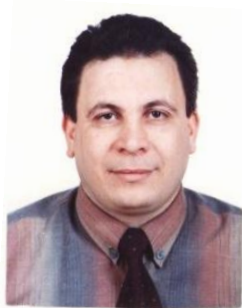

Essam Abo-Serie was born in Cairo-Egypt, 1967. He obtained BSc with honors in 1989 and MSc in 1995 from Cairo University, Mechanical Power Engineering.He completed his Ph.D. from Imperial College, Mechanical Engineering London in 2004 The major study was related to fuel injection and spray characterization using computational and laser techniques.

He was a research assistant in City University London 2002-2004.He joined Engine Lab as postdoctoral fellowat Korea Advanced Institute Science and Technology, KAIST in South Korea 2004-2005. He worked as a postdoctoral researcher at Loughborough University, UK, 2006-2007. Then he was appointed as a Senior Lecturer at Coventry University in 2007. He acted as a manager of the MSc program'Engine Design and Development' and involved in different researches and consultancy works. He then joined Cairo University in 2010 where he initiated and supervised Cairo University Formula Student Team,2012 before moving to Mevlana University in Turkey as Associate Professor and Head of Mechatronics Engineering.He has wide range of research activities in thermofluids applications both modeling and experimental. His major publications are related atomization and spray characterization fromICE injectors using different laser techniques and CFD. Healso worked and published in ground vehicle aerodynamics.

Dr. Abo-Serie is a fellow of the Higher Education Academy in UK (FHEA) and a member of Institute of Physics IOP, ILASS Europe, Egyptian society of Mechanical Engineers, Engineering Society of Mechanical Engineering ESME and a reviewer for different international journals. 